\title{
Charge measurement and mitigation for the main test masses of the GEO 600 gravitational wave observatory
}

\author{
M Hewitson ${ }^{1}$, K Danzmann ${ }^{1}$, H Grote ${ }^{1}$, S Hild ${ }^{1}$, J Hough ${ }^{2}$, H Lück ${ }^{1}$, \\ S Rowan ${ }^{2}$, J R Smith ${ }^{1}$, K A Strain ${ }^{1,2}$ and B Willke ${ }^{1}$ \\ ${ }^{1}$ Max-Planck-Institut für Gravitationsphysik (Albert-Einstein-Institut) und Universität Hannover, \\ Außenstelle Hannover, Callinstr 38, 30167 Hannover, Germany \\ ${ }^{2}$ Department of Physics and Astronomy, University of Glasgow, Glasgow, G12 8QQ, UK \\ E-mail: martin.hewitson@aei.mpg.de
}

Received 15 August 2007, in final form 5 November 2007

Published 29 November 2007

Online at stacks.iop.org/CQG/24/6379

\begin{abstract}
Spurious charging of the test masses in gravitational wave interferometers is a well-known problem. Typically, concern arises due to the possibility of increased thermal noise due to a lowering of the quality factor of modes of the test-mass suspension, or due to the potential for increased displacement noise arising from charge migration on the surface of the test masses. Recent experience gained at the GEO 600 gravitational wave detector has highlighted an additional problem. GEO 600 uses electrostatic actuators to control the longitudinal position of the main test masses. The presence of charge on the test masses is shown to strongly affect the performance of the electrostatic actuators. This paper reports on a measurement scheme whereby the charge state of the GEO 600 test masses can be measured using the electrostatic actuators. The resulting measurements are expressed in terms of an effective bias voltage on the electrostatic actuators. We also describe attempts to remove the charge from the test masses and we show that the use of UV illumination was the most successful. Using UV illumination we were able to discharge and re-charge the test masses.
\end{abstract}

PACS numbers: $95.55 . \mathrm{Ym}, 04.80 . \mathrm{Nn}$

\section{Introduction}

There are currently $6 \mathrm{~km}$ scale laser-interferometric gravitational wave (GW) detectors operational in the world: three detectors as part of the LIGO project [1]; a Japanese detector, TAMA 300 [2]; a French-Italian detector, VIRGO [3]; and a British-German collaboration, GEO 600 [4]. Together they form an international network searching for GWs from a variety of sources. All of these instruments are variations on a standard Michelson interferometer, and all use large (of the order a few kilograms) suspended optics with high-reflectivity coatings as 


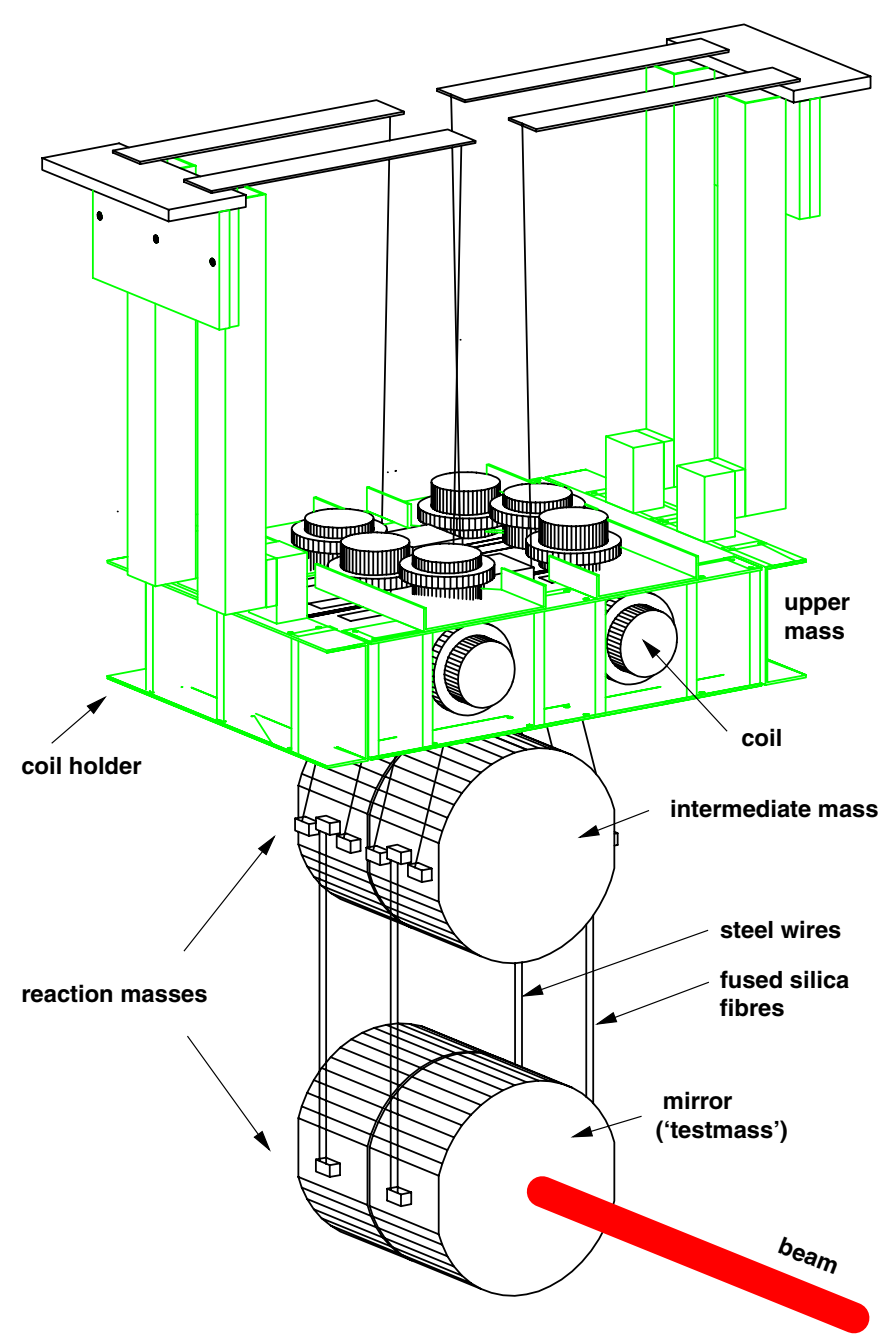

Figure 1. A schematic representation of the triple-pendulum chain from which are suspended the main test masses of GEO 600 .

the mirrors that comprise the optical cavities. The optics at the ends of the Michelson arms (whose differential length motion is detected at the dark port) are typically called test masses. In all cases, the Michelson interferometers are operated at a dark fringe and held there using actuators and active feedback control.

\section{The electrostatic actuators of GEO 600}

GEO 600 has many novel features compared to the other detectors. In the context of this paper, the most notable is the use of electrostatic drives (ESDs) as the main actuators for the test masses. The ESDs used in GEO 600 are implemented by having a gold electrode pattern deposited on the front surface of a reaction mass which is suspended behind the main test mass. A schematic of the pendulum chain is given in figure 1; a schematic of the electrode pattern coating mask is shown in figure 2 . 


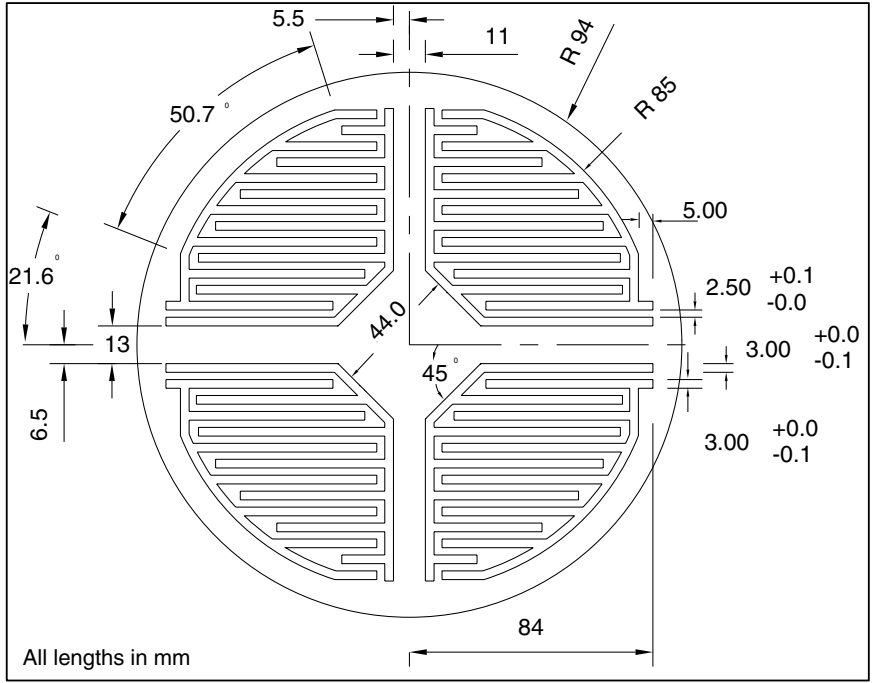

Figure 2. The design schematic for the GEO 600 ESD electrode coating mask.

By raising one electrode from each of the four pairs to a high potential while holding the other electrode of the pair to ground, an electric field is generated which can pull on the dielectric test mass. The force, $F$, exerted on the test mass can be related to the voltage applied to the electrodes by

$$
F=A V^{2}
$$

where $A$ is a constant of proportionality related to the dielectric constant and relative dielectric constant of the test mass, the spacing between the test mass and the reaction mass (about $3 \mathrm{~mm}$ ), and the geometry of the electrode pattern. For the GEO 600 ESDs, the gap between the electrodes is about $2.5 \mathrm{~mm}$, and the width of the electrodes is about $3 \mathrm{~mm}$.

In order to achieve bipolar actuation, a bias voltage, $V_{\text {bias }}$, is applied to the electrodes so that the force becomes

$$
F=A\left(V_{\text {bias }}+V_{\mathrm{s}}\right)^{2}
$$

where $V_{\mathrm{s}}$ is the actuation signal.

The displacement we achieve then depends on the bias voltage and the size of actuation signal. For frequencies well above the pendulum resonance $(f \gg 0.6 \mathrm{~Hz})$ and well below the first internal mode of the test mass $(f \ll 10 \mathrm{kHz}$ ), we can consider the test mass as a rigid and free mass. Then the displacement, $x(\omega)$, at angular frequency $\omega$ is related to the applied force by

$$
F(\omega)=-x(\omega) m \omega^{2},
$$

where $m$ is the mass of the test mass. Therefore, we can relate the displacement at angular frequency $\omega$ to the applied bias and actuation voltage. If we also set $A^{\prime}=A / m$, then

$$
x(\omega)=\frac{A^{\prime}}{\omega^{2}}\left(V_{\text {bias }}+v(\omega)\right)^{2} .
$$


If we apply an excitation signal $v\left(\omega_{0}\right)=V_{0} \cos \omega_{0}$, then

$$
\begin{aligned}
x(\omega) & =\frac{A^{\prime}}{\omega_{0}^{2}}\left(V_{\text {bias }}+V_{0} \cos \omega_{0}\right)^{2}, \\
& =\frac{A^{\prime}}{\omega_{0}^{2}}\left(V_{\text {bias }}^{2}+2 V_{\text {bias }} V_{0} \cos \omega_{0}+\frac{V_{0}^{2}}{2}\left(1+\cos 2 \omega_{0}\right)\right),
\end{aligned}
$$

where $V_{0}$ is the amplitude of the signal at angular frequency $\omega_{0}$ and the constant of proportionality, $A^{\prime}$, now incorporates the mass of the test mass. If we discard the de terms and those at $2 \omega_{0}$ (since they do not contribute to the displacement at $\omega_{0}$ ), we can write the resulting displacement as

$$
x_{\omega_{0}}=2 \frac{A^{\prime}}{\omega_{0}^{2}} V_{\text {bias }} V_{\omega_{0}},
$$

where $V_{\omega_{0}}=v\left(\omega_{0}\right)=V_{0} \cos \omega_{0}$. In the remainder of this paper we will assume that all measurements are made at a single frequency, and drop the subscript 0 notation, just writing $V_{\omega}$ and $x_{\omega}$ instead.

\section{The effect of electrical charges on the test masses of GEO 600}

In GEO 600, the test masses are suspended by fused-silica fibres from an intermediate fusedsilica mass (which is itself suspended by steel wires from an upper mass). This is done so to minimize the mechanical loss of the test mass and hence keep the energy arising from Brownian motion constrained to narrow bands of frequencies. The result is that the test masses are very well electrically isolated.

There are many mechanisms by which the test masses of GEO 600 can become charged: through venting and evacuation of the vacuum chambers (friction); by allowing the test mass to make electrical contact with the reaction mass when the electrodes are held at their nominal bias voltage; accumulating charge from cosmic-ray interactions.

Having charged-up test masses is not only bad from the point of view of noise [5-8], but it also has a strong effect on the actuation strength of electrostatic actuators which, in the case of GEO 600, means a change in the gain of the Michelson longitudinal servo, and, perhaps more importantly, a change to the absolute calibration of the main GW output which assumes constancy of the ESD actuation strength over periods of several months (after initial calibration to another reference [9]).

\section{Measuring the charge state of the test masses}

Since the signal that we apply to the ESDs is non-symmetric (one electrode biased to $+600 \mathrm{~V}$; the other held at $0 \mathrm{~V}$ ), an additional electric field is created between the reaction-mass and any surrounding metal work (for example, the vacuum tank walls); the strength of this field is proportional to the drive voltage we apply. If the test masses then become charged to some potential, they experience a force due to this additional electric field. In contrast to the dielectric action described above, the force we exert here is proportional to the actuation signal since we can both push and pull the test mass due to the charges on it. The resulting displacement produced by the electrostatic drive when the test mass is charged can then be 


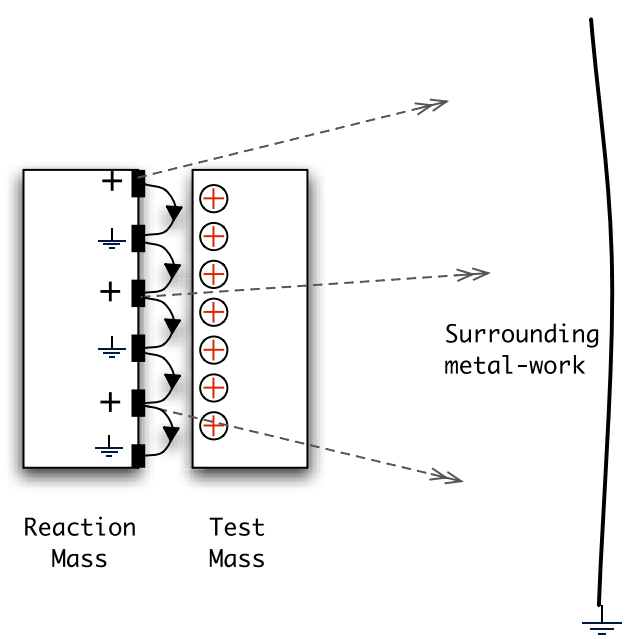

Figure 3. Schematic description of the field lines that interact with charges on the test mass. Here, the field lines emanate from the HV electrodes of the ESD and end on any extraneous metal work.

written as

$$
\begin{aligned}
x_{\omega} & =2 \frac{A^{\prime}}{\omega^{2}} V_{\mathrm{bias}} V_{\omega}+\frac{\beta}{\omega^{2}} V_{\omega} \\
& =\frac{2 A^{\prime}}{\omega^{2}}\left(V_{\mathrm{bias}}+\beta^{\prime}\right) V_{\omega},
\end{aligned}
$$

where the constants of proportionality $\beta$ and $\beta^{\prime}=\beta /\left(2 A^{\prime}\right)$ depend on the sign and amount of charge that is present on the test mass as well as its distribution, the exact form of the electric field produced by this electrode/metal-work geometry, and, to a lesser extent, on the separation of the two masses. This is depicted in figure 3.

From the right-hand side of equation (9) we can immediately see that, depending on the sign of $\beta^{\prime}$, the displacement we achieve for a given actuation signal will be either reduced or enhanced. We can think of $\beta^{\prime}$ as giving rise to an effective bias voltage, $V_{\text {eff }}$, and, as such, it is clear that there exists a value of the bias voltage, $V_{\text {bias }}=V_{\text {bias }_{0}}$, where the displacement we induce at frequency $\omega$ is zero ${ }^{3}$. This immediately suggests a method to measure a quantity that is proportional to the charge present on the test mass: for a given actuation signal, we can measure the displacement of the test mass as a function of the bias voltage. Throughout the remainder of this paper we refer to measuring the charge or the charge state of the test masses. In fact, we only ever measure something that is proportional to the charge.

\section{A summary of some charge mitigation methods}

Once we have determined that a test mass is charged, we want to remove (reduce) the charge. There are different courses of action that may achieve this, for example,

Venting. This is a practical method for discharging optical components, particularly if the backfill gas is of high humidity. However, it may be important to avoid humidity entering the system. For a negatively charged component we believe that venting is most effective if a gas of significant electron affinity, such as oxygen (in air), is used. For a positively charged

\footnotetext{
3 The bias voltage which produces no displacement is $0 \mathrm{~V}$ if the test mass is fully discharged.
} 
component we believe that the most effective approach may again be to use a gas mixture, such as air, where there are often already negatively charged ions present. Alternatively, a gas of negative electron affinity, such as argon, may be tried. We believe that if argon is used (and is effective) there is the added advantage that the components should have less tendency to charge up as the gas is pumped out of the system.

$U V$ illumination. In the context of optics for gravitational wave detectors, UV light of suitable wavelength has been shown experimentally [5] to remove electrons from the surface of dielectric components. While the physical mechanisms require detailed study this provides a method of removing excess negative charge. Alternatively the light can be shone on to a metal target near the mass and any positive charge on the mass can be neutralized by photoelectrons released from the metal. This method is employed in many experiments (see, for example, [10-13]).

Electrical contact. Electrical charge can be removed from a local area of a mass by contact with an earthed conductor. However, this method is not very effective for large and delicately suspended or mounted optical components.

\subsection{Measuring the charge on the test masses}

As described in section 4, the charge present on the test mass can be evaluated by measuring the effective displacement a given actuation signal produces as a function of the dc bias voltage applied. Simplifying equation (9) we get that the normalized (by the actuation signal) amplitude of the displacement we measure can be modelled by

$$
\bar{x}=\frac{x_{\omega}}{V_{\omega}}=\left|a V_{\text {bias }}+b\right|,
$$

where the constant $a$ can be simply related to equation (9) by $a=2 \frac{A^{\prime}}{\omega^{2}}$, and the constant $b=2 \frac{A^{\prime} \beta^{\prime}}{\omega^{2}}$. Note that the two coefficients, $a$ and $b$ depend inversely on the square of the frequency of the drive signal. We can fit this model to the measured data (by using a standard least-squares-fitting algorithm) to yield values for the two unknown coefficients. The effective bias, $V_{\text {eff }}$, due to charges on the test mass is then given by the bias voltage needed to produce zero displacement. This is just

$$
V_{\text {eff }}=-b / a \text {. }
$$

Note that $V_{\text {eff }}$ is just proportional (via the value for $\beta^{\prime}$ ) to the charge on the test mass and to the mass of the test mass.

\section{Case study: charging and discharging of the test masses of GEO 600}

During early December of 2006, a power cut at GEO 600 left the pendulums supporting the main test masses un-damped. It is believed that, at the same time, the bias voltage was still supplied to the ESDs. While the nominal gap between test mass and reaction mass is around $3 \mathrm{~mm}$, the gap between the test mass and the solder connections of the leads supplying the bias voltage to the ESD electrodes is much less, around $1 \mathrm{~mm}$. This makes it highly likely that the test mass can touch the reaction mass at these solder joints when the pendulum chain is un-damped.

After the power-cut, locking was not possible with normal parameters. After some investigation it became clear that the amount of displacement produced (for a given bias and signal voltage) by the ESDs was significantly reduced (70\% reduction for the East ESD and 


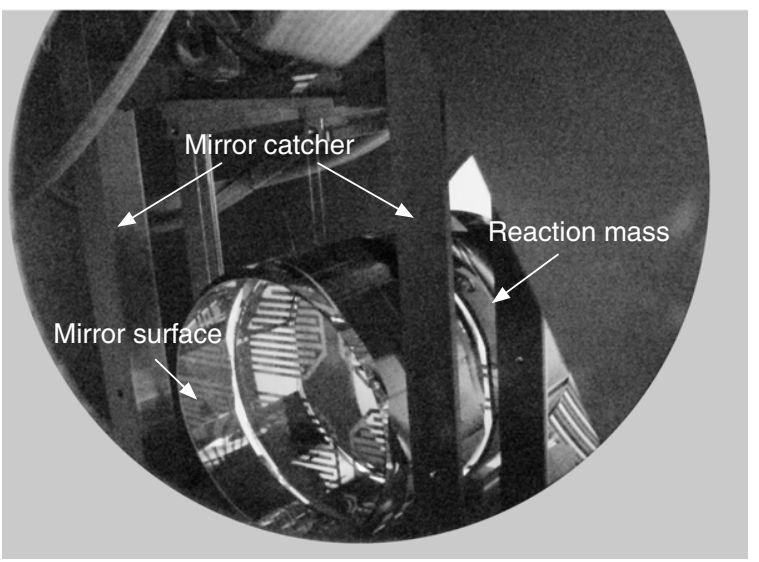

Figure 4. Photograph of the view of the East test mass as seen from the UV lamp. The mirror (test mass), reaction-mass and surrounding catcher are indicated.

$30 \%$ for the North). We then investigated this further by connecting individual quadrants of the East ESD and measured the relative actuation strengths to be $46 \%, 25 \%, 18 \%$ and $12 \%$. These measurements led to the thought that the test masses were charged-up during the power-cut by touching the solder joints.

Measuring the charge on the East test mass as described in section 5.1 gives the trace shown in figure 5. These measurements were made by injecting a signal at $696 \mathrm{~Hz}$. The fit yields values for the coefficients of $a=-8 \times 10^{-6}$ and $b=26.7 \times 10^{-4}$. From these values we get an effective bias due to charges of around $333 \mathrm{~V}$.

Two attempts were made to remove the charges from the test mass. The first attempt was to illuminate the test mass with UV light from a source ${ }^{4}$ placed external to the vacuum system and directed at the test masses through viewports on the vacuum system. The view of the test mass as seen from the UV lamp is shown in figure 4. The problem here is, at the time the relevant viewports were made from Kodial which transmits less than $2 \%$ of the incident UV. It turned out that we could not influence the charge state of the test mass after $1 \mathrm{~h}$ of illumination. From this we deduced that discharging the test mass in this way would take an unacceptably long time.

The second attempt was to flood the central vacuum cluster (which encloses the two test masses), with argon. The vacuum system was filled with about 1 mbar of argon with the ESDs switched off. After evacuating the central cluster again, we remeasured the charge state of the East test mass and saw no significant change in the charge state. We deduce that argon at the low pressure used is not effective at discharging a positively charged component. In contrast to the major components of air (nitrogen and oxygen), which have a high electron affinity and would therefore be more suitable to remove negative charges, argon, among the high purity gases we had available, has got a low ionization threshold of $15.8 \mathrm{eV}$ compared to Helium (24.6 $\mathrm{eV})$ and Neon $(21.5 \mathrm{eV})$. It had already been postulated that it may be possible at pressures around 1 mbar and, correspondingly, low breakdown voltages, that the electrical field of the charges present would sustain a short-duration glow-discharge, removing the charges from the mirror surface.

Since these mitigation efforts were not successful, we included some more invasive steps in the commissioning plan of GEO 600.

\footnotetext{
${ }^{4}$ Heraeus Noblelight: NK6-12, Mercury lamp with $800 \mathrm{~mW}$ output at $253.7 \mathrm{~nm}$.
} 


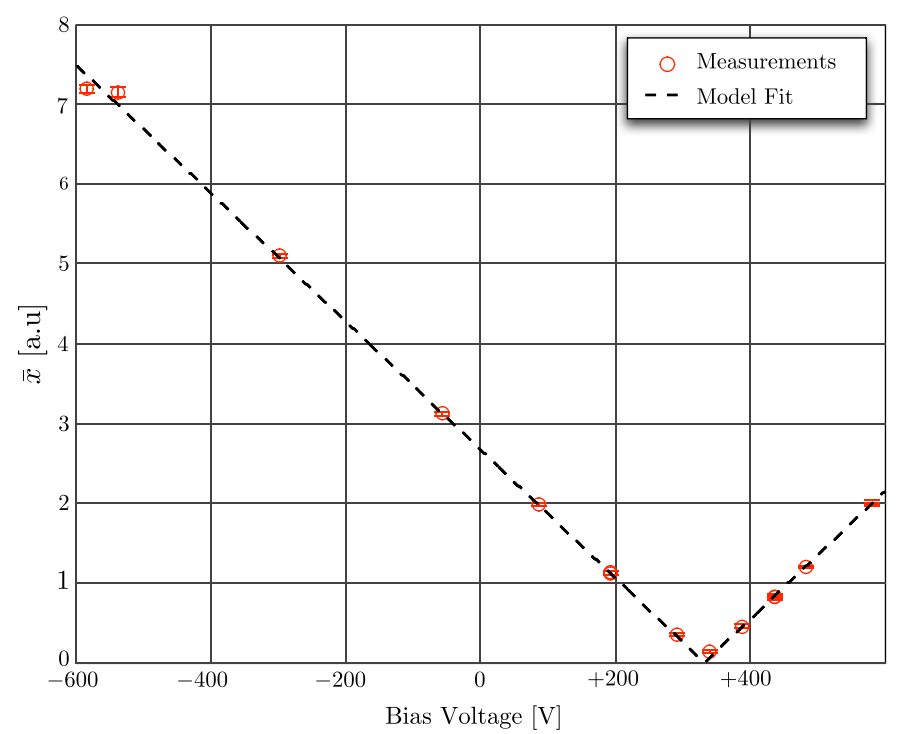

Figure 5. Measurement of the East test mass charge state after the power-cut. This measurement was made on 12 December 2006.

The commissioning plan of GEO 600 following the power-cut included opening the central cluster to re-position some beam-blocks. We decided that we would re-measure the charge state of the two test masses after this vacuum work, in the hope that venting to atmospheric pressure had removed the charges. In addition, the plan was to install fused-silica viewports which transmit close to $100 \%$ of any incident UV light. The vacuum system was opened on 21 February 2007, and following the central cluster work, the charge state of the East test mass was re-measured on 7 March 2007, this time using a signal frequency of $1234 \mathrm{~Hz}$ (this is about 85 days after the measurement shown in figure 5). The results of this measurement are shown in figure 6. The fit yields values for the coefficients of $a=-1.2 \times 10^{-6}$ and $b=1.82 \times 10^{-4}$. From these values we get an effective bias due to charges of around $148 \mathrm{~V}$. So, either the test mass was not fully discharged while being in air (room temperature around $20{ }^{\circ} \mathrm{C}$, relative humidity of about $20 \%$ ), or (more probably) the test mass was charged again during the evacuation process. It is not completely clear why the fit is poorer for this set of measurements compared to the others. One possible explanation for this is that there is an averaging process going on during this measurement. Such a process can arise if the in-homogeneous charge distribution on the test mass varies on time-scales shorter than the measurement times such that the effective zero bias is changing during the course of the measurements. One might expect charges to be able to migrate on the surface of the test mass more easily so soon after evacuating the system due to layers of gas and/or water being present on the surface of the test mass.

With the new fused-silica viewports in place, the next step was to use UV illumination again to try and discharge the test mass.

\subsection{A further attempt at discharging using UV light}

The UV source described above was used to illuminate the volume containing the East test mass, the reaction-mass and the surrounding metal work. To monitor the discharging process, we injected a sinusoidal signal into the East ESD at $1234 \mathrm{~Hz}$ and measured it at the output 


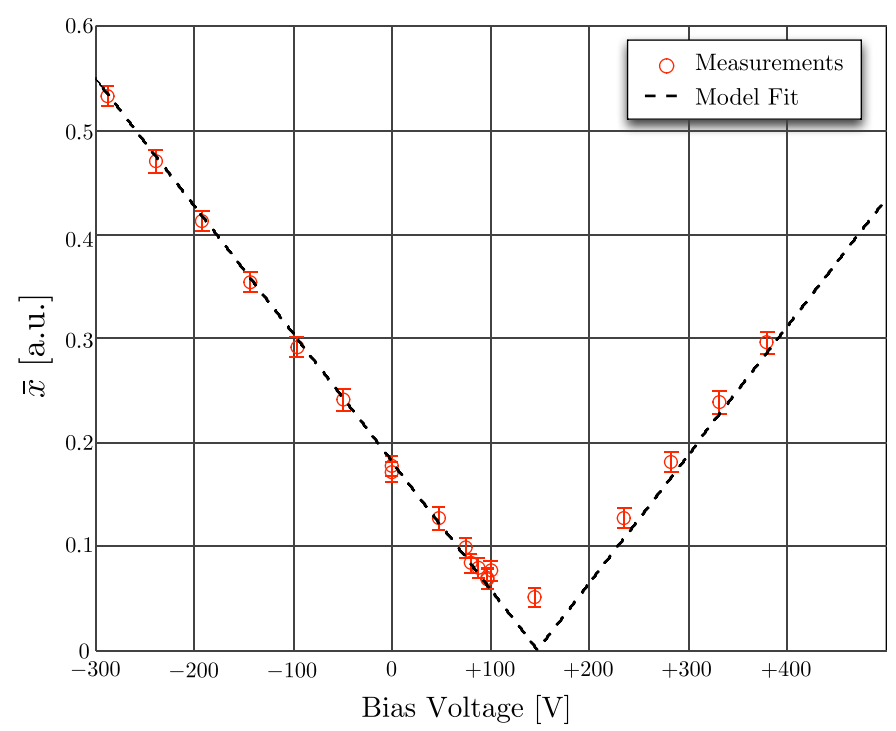

Figure 6. Measurement of the East test-mass charge state after vacuum work. This measurement was made on 7 March 2007.

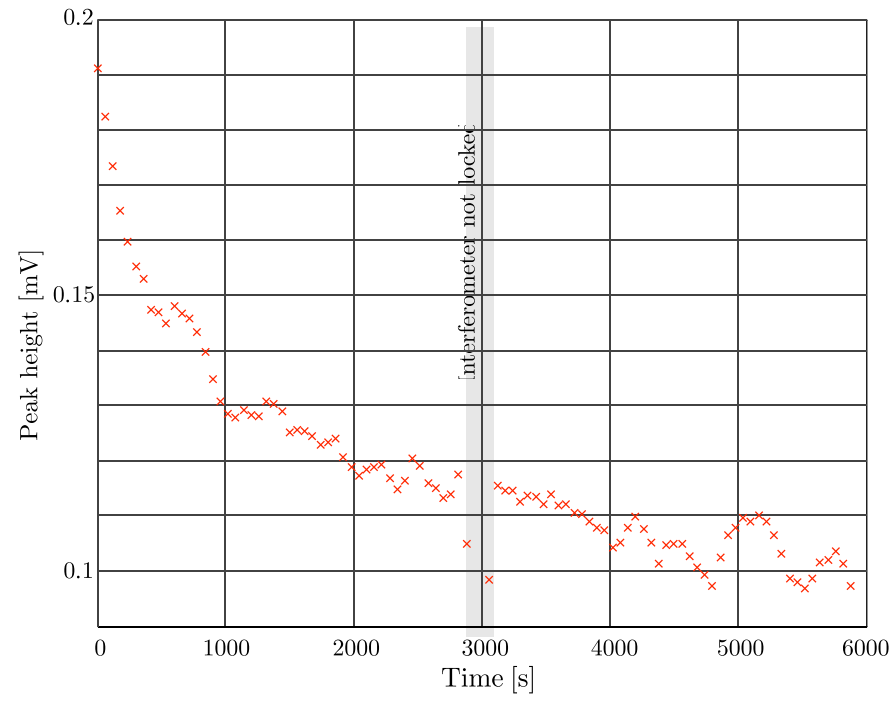

Figure 7. Tracking the actuation strength of the East ESD during the discharge process via an injected sinusoidal signal. The peak height is normalized by the power in the power-recycling cavity (i.e., the optical gain).

of the interferometer. Figure 7 shows the time-evolution of the amplitude of the injected signal measured in a spectrum of the main interferometer output signal over the first $2 \mathrm{~h}$ of the discharge process; during the period of illumination, the applied East test-mass bias was zero.

For the remaining period of illumination $(13 \mathrm{~h})$, the detector was unlocked and the bias voltage was removed from the North ESD as well, since we do not know how the electric field from the bias voltage influences the discharging process. 


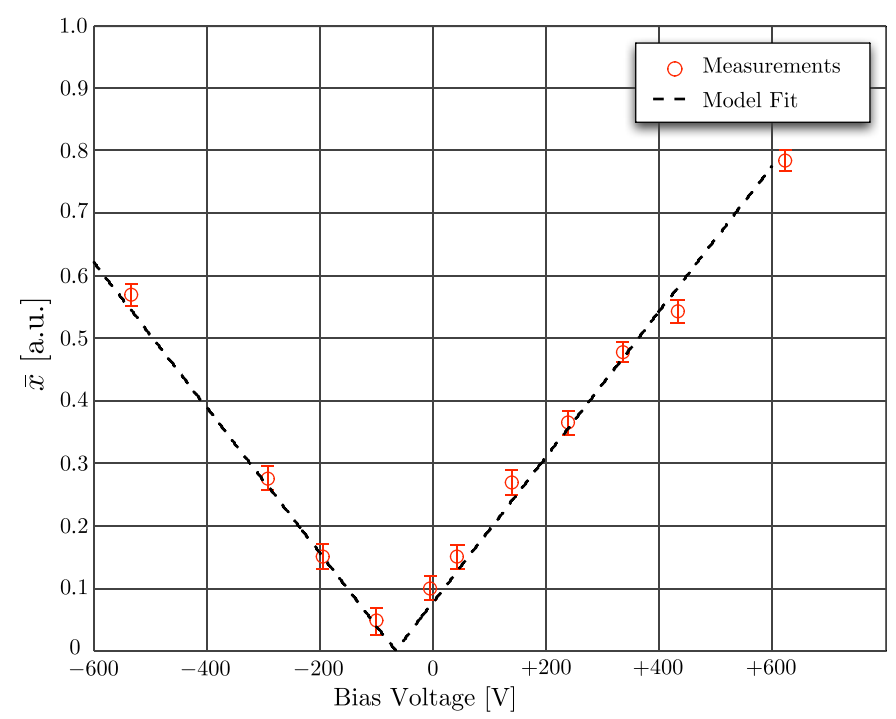

Figure 8. The charge state measurement for the East test mass after one night (around $15 \mathrm{~h}$ ) of UV illumination. The effective bias has gone from $+148 \mathrm{~V}$ to $-66 \mathrm{~V}$ in this time.

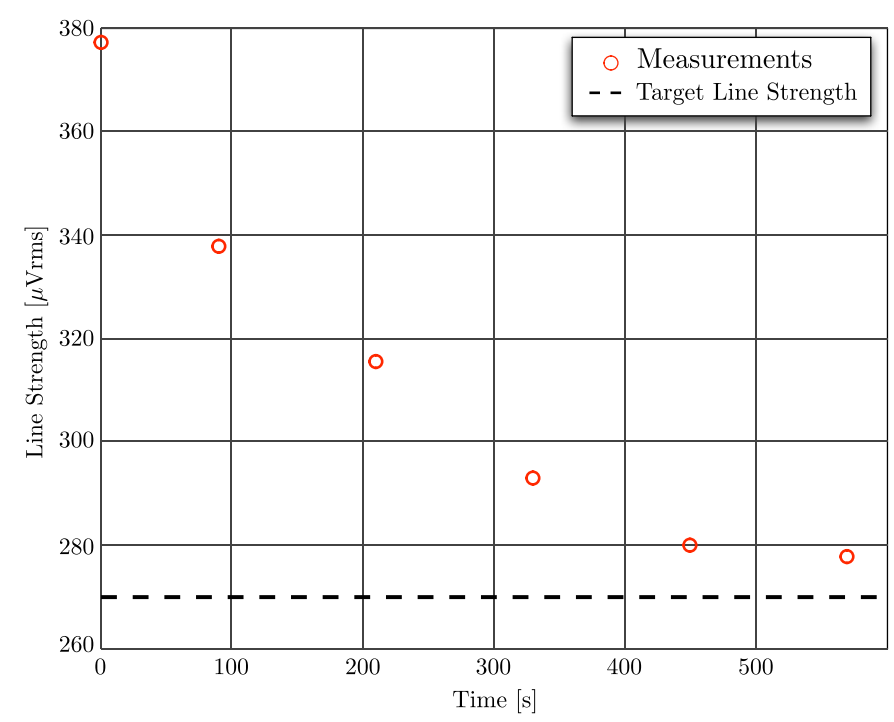

Figure 9. Measurements of the amplitude of the injected $1234 \mathrm{~Hz}$ signal during the recharging process.

Figure 8 shows the measurement of the charge state of the East test mass after overnight UV illumination (total of about $15 \mathrm{~h}$ ). The fit yields values of $a=-1.2 \times 10^{-6}$ and $b=-7.7 \times 10^{-5}$, which gives an effective bias voltage of $-66 \mathrm{~V}$. From this we can see that we have induced a negative effective bias during the illumination process. We deduce that we have released photoelectrons from the electrodes of the electrostatic drive and these have travelled to the near surface of the test mass. 


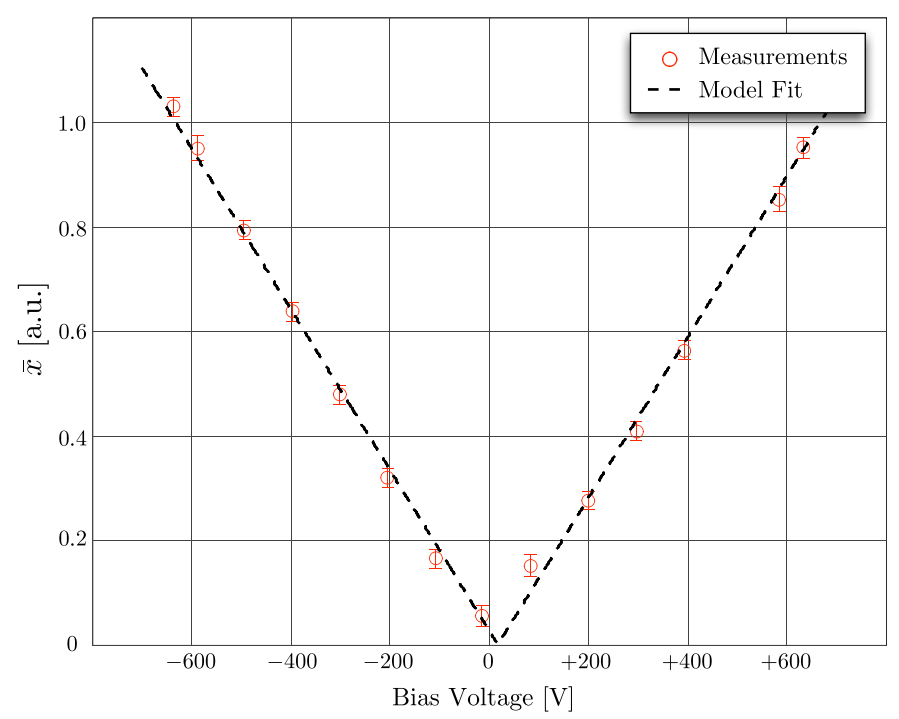

Figure 10. The charge-state measurement of the East test mass after re-charging with UV and an applied bias voltage of $+200 \mathrm{~V}$.

The next experiment was to investigate the effect of UV illumination with the bias voltage switched on, with the hope that we could re-charge the test mass to get an effective bias voltage close to zero volts.

Suspecting that this process would be very sensitive to the illumination time and the bias voltage, we set the bias voltage to $+200 \mathrm{~V}$ and decided on $20 \mathrm{~s}$ illumination periods. To monitor progress, we injected a sinusoidal signal at $1234 \mathrm{~Hz}$ into the East ESD and measured its actuation strength in the main detector output. From figure 8 it is possible to compute the target amplitude of the injected signal. At the beginning of the process, the signal amplitude was measured to be around $410 \mu \mathrm{V}_{\text {rms }}$; the target amplitude was computed to be $270 \mu \mathrm{V}_{\text {rms }}$. Figure 9 shows the individual measurements of the signal amplitude for each burst of UV.

Figure 10 shows the measurement of the charge state of the East test mass after recharging. The fit to the measurements yields values of $a=-1.5 \times 10^{-6}$ and $b=2.7 \times 10^{-5}$, which gives an effective bias voltage of $17.6 \mathrm{~V}$. In this case, we deduce that we have liberated electrons from the surface of the test mass and these have migrated to the appropriate electrode of the electrostatic drive under the applied electric field.

\section{Concluding remarks}

Table 1 summarizes the measurements presented in this paper. The errors on the parameters are estimated by assuming a Gaussian noise model for the errors and by taking the noise-floor surrounding the measurements as a measure of the error on the peak amplitudes. We then assume that the errors on $a$ and $b$ could be maximally correlated such that the maximum error on $V_{\text {eff }}$ is just taken as the sum of these two errors.

Note that the values for $a$ in the fits should be independent of the charge and hence may be expected to be the same for all measurements made at the same frequency. However, they do depend on the gap between the test mass and reaction mass, and hence this value can fluctuate over time. In addition, the optical gain of GEO 600 is frequency dependent, so measurements made at different frequency not only scale by $1 / \omega^{2}$, but also by the optical gain 
Table 1. Summary of charge-state measurements made during the various experiments described throughout this paper.

\begin{tabular}{|c|c|c|c|c|}
\hline Value of $a$ & Value of $b$ & $V_{\text {eff }}[\mathrm{V}]$ & $\chi^{2} /$ n.d.o.f & Measurement details \\
\hline$-8 \times 10^{-6} \pm 0.1 \%$ & $26.7 \times 10^{-4} \pm 0.2 \%$ & $333 \pm 1$ & 15 & $\begin{array}{l}\text { Measurement made after power-cut: } \\
12 \text { December } 2006 . \text { Measurement } \\
\text { frequency was } 696 \mathrm{~Hz} \text {. }\end{array}$ \\
\hline$-1.2 \times 10^{-6} \pm 1 \%$ & $1.82 \times 10^{-4} \pm 1.2 \%$ & $148 \pm 3$ & 4 & $\begin{array}{l}\text { Measurement made after vacuum work: } \\
7 \text { March } 2007 \text {. Measurement frequency } \\
\text { was } 1234 \mathrm{~Hz} \text {. }\end{array}$ \\
\hline$-1.2 \times 10^{-6} \pm 1 \%$ & $-7.7 \times 10^{-5} \pm 4 \%$ & $-66 \pm 3$ & 7 & $\begin{array}{l}\text { Measurement made after overnight UV } \\
\text { illumination. Measurement frequency } \\
\text { was } 1234 \mathrm{~Hz} \text {. }\end{array}$ \\
\hline$-1.5 \times 10^{-6} \pm 0.5 \%$ & $2.7 \times 10^{-5} \pm 9 \%$ & $17.6 \pm 2$ & 5 & $\begin{array}{l}\text { Measurement made after re-charging process } \\
\text { with applied bias and UV illumination. } \\
\text { Measurement frequency was } 1234 \mathrm{~Hz} \text {. }\end{array}$ \\
\hline
\end{tabular}

transfer function. This is the reason that the first measurement has a different value of $a$, since it was made at a different frequency. If we scale $a$ from the first experiment by the square of the ratio of the frequencies $\left(696^{2} / 1234^{2}\right)$ and by the ratio of the optical gain at these two frequencies $(0.6 / 1.5)$, we get a value of $a=-1.0 \times 10^{-6}$ which we can directly compare to the other measurements.

Our results presented here clearly show the efficacy of employing a UV light source for charge mitigation in an operating long-baseline gravitational wave interferometer. Since GEO 600 is an operational GW observatory, all detector investigations have to be carefully weighed for risk against damage and sensitivity reduction. As such, further charge experiments are not permitted on the instrument once the charge state of the test masses is returned to effectively zero. However, there remain a number of open questions related to the use of UV for charge mitigation in interferometric detectors which require additional investigation and can be pursued in the laboratory. High-fluence UV laser pulses are known to cause damage in fused silica, hence studies of the effect of UV under similar conditions (wavelength, power, duration of illumination) used here on the optical properties of the substrates and coatings are of particular interest and under investigation in the laboratory [14]. In addition, for planned future generations of GW detectors, the implementation of methods of preventing the initial build-up of charge on the optics would be desirable. Thus, the appropriateness of various techniques is currently being assessed, including the use of thin coatings of conductive tin oxide [15] applied to the test masses and/or suspensions. The mechanical dissipation and optical absorption of such coatings to determine their suitability for this purpose is a subject of ongoing study.

\section{Acknowledgments}

The authors are grateful for support from PPARC, the Leverhulme Trust and the University of Glasgow in the UK, and the BMBF and the state of Lower Saxony in Germany. This document has been assigned LIGO Laboratory document number LIGO-P070087-00-Z.

\section{References}

[1] Waldman S J (for the LIGO Science Collaboration) 2006 Status of LIGO at the start of the fifth science run Class. Quantum Grav. 23 S653-60 
[2] Ando M and (the TAMA Collaboration) 2005 Current status of the TAMA300 gravitational-wave detector Class. Quantum Grav. 22 S881-9

[3] Acernese F et al 2006 The Virgo status Class. Quantum Grav. 23 S635-42

[4] Hild S (for the LIGO Scientific Collaboration) 2006 The status of GEO 600 Class. Quantum Grav. 23 S643-51

[5] Rowan S, Twyford S, Hutchins R and Hough J 1997 Investigations into the effects of electrostatic charge on the Q factor of a prototype fused silica suspension for use in gravitational wave detectors Class. Quantum Grav. 14 1537-41

[6] Mitrofanov V, Prokhorov L, Tokmakov K and Willems P 2004 Investigation of effects associated with variation of electric charge on a fused silica test mass Class. Quantum Grav. 21 S1083-9

[7] Sumner T, Araújo H, Davidge D, Howard A, Lee C, Rochester G, Shaul D and Wass P 2004 Description of charging/discharging processes of the LISA sensors Class. Quantum Grav. 21 S597-602

[8] Jafry Y, Sumner T J and Buchman S 1996 Electrostatic charging of space-borne test bodies used in precision experiments Class. Quantum Grav. 13 A97-A106

[9] Hewitson M, Heinzel G, Smith J R, Strain K A and Ward H 2004 Principles of calibrating the dual-recycled GEO 600 Rev. Sci. Instrum. 754702

[10] Sun K-X, Allard B, Buchman S, Williams S and Byer R L 2006 LED deep UV source for charge management of gravitational reference sensors Class. Quantum Grav. 23 S141-50

[11] Buchman S, Quinn T, Keiser G M, Gill D and Sumner T J 1995 Charge measurement and control for the gravity probe B gyroscopes Rev. Sci. Instrum. 66120

[12] Wass P J et al 2006 Testing of the UV discharge system for LISA Pathfinder AIP Conf. Proc. 873220

[13] Schulte M, Rochester G K, Shaul D N A, Sumner T J, Trenkel C and Wass P J 2006 The charge-management system on LISA-pathfinder-status and outlook for LISA AIP Conf. Proc. 873165

[14] Sun K-X et al 2007 Update of LIGO Test Mass Charging Mitigation Activities at Stanford LIGO document no: G070208-00-Z http://admdbsrv.ligo.caltech.edu/dcc/

[15] Batzill M and Diebold U 2005 The surface and materials science of tin oxide Prog. Surf. Sci. 79 47-154 